\title{
Circulating Tumor Cells Were Associated with the Number of T Lymphocyte Subsets and NK Cells in Peripheral Blood in Advanced Non-Small-Cell Lung Cancer
}

\author{
Liang Ye, ${ }^{1,2}$ Fang Zhang, ${ }^{3}$ Huijuan $\mathrm{Li}^{2}{ }^{2}$ Linfei Yang, ${ }^{1}$ Tangfeng $\mathrm{Lv},{ }^{3}$ Wei Gu, ${ }^{1}$ and Yong Song ${ }^{2}$ \\ ${ }^{1}$ Department of Respiratory Medicine, Nanjing First Hospital, Nanjing Medical University, Nanjing 210006, China \\ ${ }^{2}$ Department of Respiratory Medicine, Jinling Clinical Medical College of Nanjing Medical University, Nanjing 210006, China \\ ${ }^{3}$ Department of Respiratory Medicine, Jinling Hospital, Nanjing University School of Medicine, Nanjing 210006, China
}

Correspondence should be addressed to Wei Gu; guwei_101@163.com and Yong Song; yong_song6310@yahoo.com

Received 5 April 2017; Accepted 25 October 2017; Published 24 December 2017

Academic Editor: Alvaro González

Copyright (@) 2017 Liang Ye et al. This is an open access article distributed under the Creative Commons Attribution License, which permits unrestricted use, distribution, and reproduction in any medium, provided the original work is properly cited.

\begin{abstract}
In this study, we aim to investigate the correlation between circulating tumor cells (CTCs) and the T lymphocyte subsets and NK cells in peripheral blood in non-small-cell lung cancer (NSCLC). The peripheral blood CTCs were determined by SET-iFISH. Flow cytometry was used to determine the distribution of T lymphocyte subsets and NK cells. Forty-one (49\%) patients showed positivity for CTCs. Logistic regression analysis revealed CTC number was negatively correlated with the ratio of CD3+, CD4+, CD4+/CD8+, and NK \% in patients at stage IV, while in a positive correlation was noticed between CTC number and regulatory T cell (Tregs) ratio in these patients. Multivariate analysis was performed in combination with the clinical-pathological materials to identify the risk factors for CTC positivity. Differentiation, NSCLC stage, percentages of CD3+CD4+ cells, Tregs, and NK cells were the independent risk factors for CTCs. CTCs were associated with the decrease of immune surveillance in the peripheral blood in NSCLC patients. The decrease of immune surveillance contributed to the escape of CTCs from the killing effects of the immunocytes, as well as the formation of metastasized lesions in the target organs.
\end{abstract}

\section{Introduction}

Lung cancer is the leading cause of cancer-related mortality worldwide. Most of the lung cancer patients usually die from recurrence and distal metastasis [1-3]. Nowadays, the management of lung cancer is still a challenge. Blood dissemination is the major cause for metastasis of lung cancer, through which distal metastasis is achieved with blood circulation [4, 5]. Circulating tumor cells (CTCs) represent a heterogeneous population of malignant cells that disseminate into the blood circulation, escaping from the immune surveillance in the presence of various cytokines. Under certain conditions, these cells could enter the target organs and contribute to the metastasis of cancer cells. On this basis, CTCs are considered as a prognostic factor in primary and metastatic cancers $[6,7]$.

Cancer cells have been detected in the peripheral blood in the patients with solid tumor, and the CTCs are considered as the origin for the metastasis and recurrence. Besides, CTC enumeration offers potential utility as a prognostic biomarker and predictor, and it may fulfill the criteria for a surrogate response biomarker $[8,9]$.

In the past decades, the immune system was reported to inhibit the progression of cancer cells [10-12]. In healthy individuals, a large number of immunocytes are detected in the peripheral blood, including T lymphocytes, NK cells and B lymphocytes, which play crucial roles in the immune surveillance, immunosuppression and killing effects. Such process is considered to be highly related to the CTCs [13-15]. For example, cytotoxic sinusoidal lymphocytes of liver transplant were reported to be effective against CTCs [16]. Cancer patients were usually in an immunocompromised state, together with a decrease of immunocytes in the peripheral blood. On this basis, CTCs may escape the immune response and access to the target organ through blood circulation, which contributed to the metastasis. 


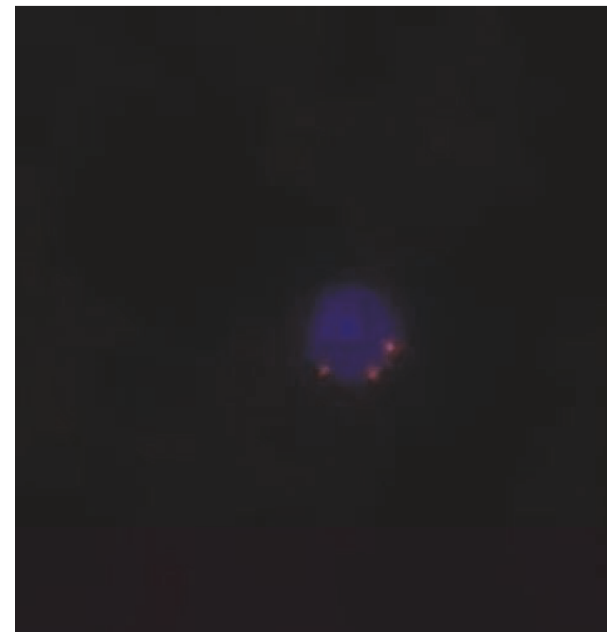

FIGURE 1: Immunostaining of a single lung cancer CTC isolated from patient peripheral blood. The CTCs were observed through combining CD45, DAPI, and fluorescence in situ hybridization with the centromere of chromosome 8 (CEP8). CD45-DAPI+ CEP8 $>2$ was considered as CTC.

To date, rare studies have been focusing on the correlation between CTCs and the distribution of immunocytes in peripheral blood. In a previous study, Mego et al. [17] reported that CTCs were associated with the defect of adaptive immunity in breast cancer patients. In this study, we aim to investigate the correlation between CTCs and T lymphocyte subsets in the peripheral blood in non-small cell lung cancer (NSCLC) patients.

\section{Materials and Methods}

2.1. Patients. Eighty-three late-stage primary NSCLC patients admitted to our hospital from November 2013 to January 2015 were included in this study. NSCLC was confirmed using pathological analysis, and the staging of the late-stage NSCLC (IIIa, IIIb, and IV) was carried out according to the seventh edition of the American Joint Committee on Cancer (AJCC) staging manual. Clinical data including age, ethnicity, histological subtype, smoking status, and sites of metastasis were collected. The inclusion criteria were as follows: those with a WHO Performance Status of 0-2; those who received no radiotherapy or chemotherapy before; and those with an expected survival of $>3$ months. The exclusion criteria were as follows: those with a history of malignancy within 5 years and those with severe disorders or complications (e.g., heart failure). Thirty-five healthy individuals received physical examinations in our hospital served as healthy control. Written informed consent was obtained from each subject. The study protocols were approved by the Ethics Committee of Nanjing First Hospital, Nanjing Medical University.

2.2. Detection of CTCS by SET-iFISH. The enrichment of CTCs was carried out according to the previous description $(18,19)$ with slight modifications. Briefly, peripheral blood ( $4 \mathrm{ml}$ ) was treated using lysis of red blood cells. Afterwards, the pellets were resuspended in PBS buffer followed by incubating with anti-CD45 monoclonal antibody-coated magnetic beads for $30 \mathrm{~min}$. The mixture was separated by magnetic beads using a magnetic stand (Promega, Madison, WI, USA). Enriched lung cancer CTCs were identified using CD45-FISH as previously described [16]. The CEP8 probe and specimen were hybridized in DAKO at $37^{\circ} \mathrm{C}$ for $20 \mathrm{~min}$ and then washed in $50 \%$ formamide at $43^{\circ} \mathrm{C}$ for $15 \mathrm{~min}$. Finally, the specimens were washed with $0.2 \%$ BSA, followed by incubating with the CD45 mixture/2\% BSA conjugated to Alexa Fluor 594 (Invitrogen) for $1 \mathrm{~h}$. The specimens were then stained with DAPI, and the fixed sample should be observed entirely under a microscope (Nikon). Positive CTCs were defined as hyperdiploid CEP8+/DAPI+/CD45- (Figure 1). The CTC count of $>2$ in $4 \mathrm{~mL}$ blood was considered positive.

2.3. Analysis of T Lymphocyte Subsets in Peripheral Blood. Peripheral blood mononuclear cells (PBMCs) were isolated using the Ficoll-Paque (Pharmacia) density-gradient centrifugation. After blocking with FcR, PBMCs were incubated with diluted antibodies for phenotyping and then analyzed using flow cytometry (FC-500, Beckman Coulter, FL, USA). Data analysis was conducted using FlowJo software (version 7.6.2, Tree Star, Ashland, OR, USA). Circulating T lymphocyte subgroup and NK cells were identified by multiparameter flow cytometry according to the previous description $[18,19]$. T lymphocyte subsets were identifying CD4FITC/CD8-PE/CD3-PC5, and NK cell was identifying with CD3-FICT/CD(16+56)-PE (BD Biosciences, CA, USA). The phenotype of NK cells was CD3-CD16+CD56+. Four-color flow cytometry (FC-500, Beckman Coulter, FL, USA) was performed to determine the phenotypes of $\mathrm{T}$ regulatory cells (Tregs) using the CD3-PC5, CD4-PE, CD25-FITC (BD Biosciences, CA, USA), and CD127-PC7 antibodies (BioLegend, San Diego, CA, USA). Tregs were defined as CD3+CD4+CD25+CD127- cells.

2.4. Statistical Analysis. The data are expressed as means \pm standard error of the mean (SEM) unless specified. The association between CTCs and individual clinical characteristics (e.g., stage, PS, histology, smoking, and sites of metastasis) was compared by Fisher's exact test. Normality of distribution was tested by the Kolmogorov-Smirnov test. If data were normally distributed, analysis of variance was used for the intergroup comparison. For data with not normally distributed, the equivalent nonparametric tests were performed and data were expressed as median (interquartile range). A least significant difference (LSD) test was used for paired comparison between two groups in presence of statistical significance after comparison among multiple groups. Student's $t$-test was used to compare the parameters between immune cells and CTC status. Univariate analysis was depicted using Pearson's coefficient. A multilinear regression analysis was performed with immune cells, tumor grade (1 and 2 versus 3 ), and tumor stage (stages IIIa and IIIb IV versus stage IV) as the independent variables and CTCs as the dependent variable. $P<0.05$ was considered statistically significant. 
TABLE 1: Relationship between the presence of circulating tumor cells (CTCs) and the clinical features of non-small-cell lung cancer.

\begin{tabular}{|c|c|c|c|c|c|}
\hline Clinical features & Number of cases & CTC-positive (\%) & CTC-negative (\%) & $x^{2}$ & $P$ values \\
\hline \multicolumn{6}{|l|}{ Age } \\
\hline$\leq 60$ years old & 23 & $15(65.2)$ & $8(34.8)$ & 3.186 & 0.18 \\
\hline$>60$ years & 60 & $26(43.3)$ & $34(56.7)$ & & \\
\hline \multicolumn{6}{|l|}{ Gender } \\
\hline Male & 48 & $24(50.0)$ & $24(50.0)$ & 0.017 & 0.90 \\
\hline Female & 35 & $17(48.6)$ & $18(51.4)$ & & \\
\hline \multicolumn{6}{|l|}{ Smoking } \\
\hline Yes & 36 & $16(61.5)$ & $20(38.5)$ & 0.474 & 0.49 \\
\hline No & 47 & $25(53.2)$ & $22(46.8)$ & & \\
\hline \multicolumn{6}{|l|}{ Histological features } \\
\hline Adenocarcinoma & 35 & $17(48.6)$ & $18(51.4)$ & 0.184 & 0.91 \\
\hline Squamous cell carcinoma & 41 & $20(48.8)$ & $21(51.2)$ & & \\
\hline Others & 7 & $4(57.1)$ & $3(42.9)$ & & \\
\hline \multicolumn{6}{|l|}{ Tumor grade } \\
\hline High differentiation & 17 & $5(29.4)$ & $12(70.6)$ & 11.183 & 0.04 \\
\hline Moderate differentiation & 36 & $14(38.9)$ & $22(61.1)$ & & \\
\hline Poor differentiation & 30 & $22(73.3)$ & $8(26.7)$ & & \\
\hline \multicolumn{6}{|l|}{ Pathological stage } \\
\hline IIIA & 14 & $2(14.3)$ & $12(85.7)$ & 18.129 & 0.00 \\
\hline IIIB & 17 & $4(23.5)$ & $13(76.5)$ & & \\
\hline IV & 52 & $35(49.4)$ & $17(50.6)$ & & \\
\hline
\end{tabular}

\section{Results}

3.1. Demographic Information. Eighty-three late-stage NSCLC patients were included in this study, among whom 14 were of IIIa stage, 17 of IIIb stage, and 52 of IV stage, respectively. Compared with the healthy individuals, no statistical difference was noticed in the age and sex ratio in the late-stage NSCLC patients $(P>0.05$, Table 1$)$.

3.2. Association between CTCs and NSCLC. Forty-one (49\%) patients showed positivity for CTC. No correlation was noticed between CTC enumeration and the histological features $(P>0.05$, Table 1$)$. The CTC number showed no statistical difference in patients aged $\leq 60$ yrs compared with those aged $>60$ yrs $(P>0.05)$. Whereas, higher CTC number was correlated with the pathological stages $(P<0.05)$. To be exact, the number of CTC in the patients of the IV stage was significantly higher than that of the counterparts of IIIa and IIIb, respectively. Besides, the CTC-positive rate was statistically correlated with the differentiation of cancer cells $(P<0.05)$.

3.3. Alternations of T Lymphocyte Subsets and NK Cells in Late-Stage NSCLC Patients. Compared with the healthy control, the ratio of $\mathrm{CD} 3+, \mathrm{CD} 3+\mathrm{CD} 4+$, and $\mathrm{NK}$ cells was decreased in the $\mathrm{T}$ lymphocytes in the peripheral blood of patients with late-stage NSCLC $(P<0.05$, Table 2$)$. Besides, the ratio of Tregs was significantly elevated in the latestage NSCLC patients compared with the healthy control $(P<0.05)$. Compared with the IIIa or IIIb NSCLC patients, a significant decrease was observed in the ratio of $\mathrm{CD} 3+$,
CD3+CD4+, CD4+/CD8+, and NK cells in those with at the IV stage. Besides, significant elevation was observed in the Tregs ratio in the patients at IV stage compared with those at IIIa or IIIb. No statistical difference was noticed in the T lymphocyte subsets between the NSCLC patients at IIIa and IIIb stages $(P>0.05)$.

3.4. Correlation between CTCs and T Lymphocyte Subsets and NK Cells. Univariate analysis showed the percentages of $\mathrm{CD} 3+, \mathrm{CD} 4+$, and $\mathrm{NK}$ cells in CTC-positive patients were significantly decreased compared with those of the CTCnegative patients. In contrast, the ratio of Tregs in CTCpositive patients was obviously higher than that of the CTC-negative patients $(P<0.05$, Table 3$)$. Logistic regression analysis revealed CTC number was negatively correlated with the ratio of $\mathrm{CD} 3+, \mathrm{CD} 4+, \mathrm{CD} 4+/ \mathrm{CD} 8+$, and $\mathrm{NK} \%$ in patients at stage IV, while in a positive correlation was noticed between CTC number and Tregs ratio in these patients (Table 4). Furthermore, multivariate analysis was performed in combination with the clinical-pathological materials to identify the risk factors for CTC positivity. As revealed in Table 5, differentiation, NSCLC stage, and percentages of CD3+CD4+ cells, Tregs, and NK cells were the independent risk factors for CTCs.

\section{Discussion}

In line with the previous study [9], we testified the presence of CTCs in the peripheral blood in the late-stage NSCLC patients. For the first time, our study revealed 
TABLE 2: Association between NSCLC and different subpopulations of T cells (mean \pm SD).

\begin{tabular}{|c|c|c|c|c|c|c|}
\hline $\begin{array}{l}\text { Variables } \\
(\%)\end{array}$ & $\begin{array}{c}\text { Control } \\
n=35\end{array}$ & $\begin{array}{c}\text { Stage IIIa } \\
n=14\end{array}$ & $\begin{array}{c}\text { Stage IIIb } \\
n=17\end{array}$ & $\begin{array}{c}\text { Stage IV } \\
n=52\end{array}$ & $F$ value & $P$ value \\
\hline $\mathrm{CD} 3+$ & $71.7 \pm 2.5$ & $69.8 \pm 1.6^{*}$ & $69.7 \pm 1.8^{*}$ & $65.7 \pm 2.7^{* \dagger \S}$ & 46.7 & 0.00 \\
\hline $\mathrm{CD} 4+$ & $39.4 \pm 0.9$ & $37.0 \pm 1.2^{*}$ & $36.9 \pm 0.9^{* \dagger}$ & $31.7 \pm 3.5^{* \S}$ & 74.4 & 0.00 \\
\hline CD8+ & $29.2 \pm 0.7$ & $29.3 \pm 0.9$ & $29.6 \pm .9$ & $29.5 \pm 1.1$ & 0.79 & 0.61 \\
\hline CD4+/CD8+ & $1.4 \pm 0.1$ & $1.3 \pm 0.1^{*}$ & $1.3 \pm 0.1^{*}$ & $1.1 \pm 0.1^{* \dagger \S}$ & 106.5 & 0.00 \\
\hline $\mathrm{NK}$ & $18.1 \pm 1.2$ & $15.8 \pm 0.9^{*}$ & $15.7 \pm 0.8^{*}$ & $14.6 \pm 1.2^{* \dagger \varsigma}$ & 70.4 & 0.00 \\
\hline Treg cell & $5.4 \pm 0.4$ & $7.4 \pm 0.5$ & $8.0 \pm 1.0$ & $8.9 \pm 1.6$ & 63.2 & 0.01 \\
\hline
\end{tabular}

${ }^{*} P<0.05$, versus control; ${ }^{\dagger} P<0.05$, versus stage IIIa; ${ }^{\circledR} P<0.05$, versus stage IIIb.

TABLE 3: Univariate analysis between CTC count (per $4.5 \mathrm{~mL}$ peripheral blood) and different subpopulations of $\mathrm{T}$ cells.

\begin{tabular}{lcccc}
\hline Variables & CTC-positive & CTC-negative & $T$ value & $P$ value \\
\hline CD3+ & $64.9 \pm 2.4$ & $69.2 \pm 2.1$ & 8.78 & 0.041 \\
CD4+ & $30.3 \pm 2.8$ & $36.5 \pm 1.7$ & 12.40 & 0.003 \\
CD8+ & $29.2 \pm 0.9$ & $29.7 \pm 1.0$ & 2.29 & 0.173 \\
CD4+/CD8+ & $1.0 \pm 0.1$ & $1.2 \pm 0.8$ & 11.07 & 0.235 \\
NK & $14.2 \pm 0.9$ & $15.7 \pm 0.9$ & 7.13 & 0.026 \\
Treg cell & $9.4 \pm 1.2$ & $7.6 \pm 1.0$ & -7.07 & 0.004 \\
\hline
\end{tabular}

TABle 4: Correlation between CTC count and different subpopulations of $\mathrm{T}$ cells.

\begin{tabular}{lcc}
\hline Parameters & Correlation coefficient & $P$ value \\
\hline CD3+ & -0.735 & $<0.001$ \\
CD4+ & -0.716 & $<0.001$ \\
CD8+ & 1.002 & 0.075 \\
CD4+/CD8+ & -0.943 & 0.061 \\
NK & -0.648 & $<0.001$ \\
Treg cell & 0.631 & $<0.001$ \\
\hline
\end{tabular}

TABLE 5: Multivariate logistic regression of the CTC positive model.

\begin{tabular}{lcccc}
\hline Variables & Odds ratio & 95\% CI low & 95\% CI upper & $P$ value \\
\hline \% CD3+ & 0.65 & 0.43 & 0.98 & 0.031 \\
\% CD3+CD4+ & 0.96 & 0.75 & 1.03 & 0.043 \\
\% Treg & 2.36 & 1.21 & 6.34 & 0.027 \\
\% NK & 0.86 & 0.75 & 0.95 & 0.012 \\
Grade & 9.73 & 1.46 & 30.25 & 0.032 \\
Stage & 32.46 & 1.15 & 367.24 & 0.001 \\
\hline
\end{tabular}

the correlation between lymphocyte subsets and CTCs in the peripheral blood of NSCLC patients, which implied a close relationship between the decrease of peripheral immune surveillance and CTCs. Such process was featured by a decreased ratio of NK cells, CD3+, CT4+, and T cells, as well as elevation of the Treg ratio. The immune disorder contributed to the dissemination of cancer cells into the circulation that escaped the killing effects of the immunocytes and formation of metastasis after migrating to the target organs, which finally resulted in disease progression and a poor survival rate.

Up to now, several methods have confirmed the presence of CTCs in NSCLC patients, especially the late-stage patients. Besides, a correlation was established between the CTC number and the clinical staging of the disease, which was considered to play important roles in the early screening, efficiency monitoring, and prognosis evaluation, as well as preparation of regimen for the individual therapy [20-23]. In this study, we determined the CTC number in peripheral blood using the SET-iFISH in 83 cases with late-stage NSCLC, among which 41 (49\%) showed positivity for CTC screening. Besides, the number of CTCs in the patients at stage IV was obviously higher than those at stage III. In line with the previous study [24], no CTCs were detected in the peripheral blood of the healthy individuals. Meanwhile, we also analyzed the correlation between CTC-positive rate and the clinical-pathological features of the late-stage NSCLC patients, which demonstrated clinical staging, differentiation of cancer cells, and distal metastasis were the risk factors for CTC positivity.

As is known to all, abundant immunocytes were presented in the peripheral blood, including T lymphocytes, B lymphocytes, and NK cells. These cells could kill the cancer cells that desquamated into the peripheral blood [25-28]. CTCs had been detected in the peripheral blood in patients with lung cancer; however, the exact mechanism of how CTCs escape from the killing effects of the immunocytes is still not well defined.

Increasing evidence shows the immune status is closely related to the pathogenesis and development of cancer in hosts [29-31]. Under normal conditions, the interaction between the $\mathrm{T}$ cell subsets is crucial for the immune function in the individuals. Whereas, in cases of aberrant alternations in the quantity and function of the $\mathrm{T}$ cell subsets, the cancer cells may escape from the immune attack in the presence of immune dysfunction and pathological changes. To our best knowledge, $\mathrm{T}$ cell subsets consist of various subsets with different functions, among which CD3+ cells are defined as the total $\mathrm{T}$ lymphocytes representing the whole immune status including CD4+ and CD8+ cells. NK cells play important roles in the antitumor immunity and inhibition of the dissemination of malignant cancer cells. These cells could kill the cancer cells through secreting cytotoxic cytokines in the absence of sensitization, which contributed to the prevention 
of cancer invasion and metastasis and finally prevented the early dissemination of cancer cells. Tregs, as a T lymphocyte subset that could negatively modulate the immune function, could inhibit the development and differentiation of the effector cells that could recognize the cancer cells. On this basis, Treg was considered to closely participate in the immunological tolerance of cancer cells. Recently, extensive studies have indicated elevation of Tregs in the tumor microenvironment or peripheral blood as a predictor for progression and poor prognosis $[28,32]$. It has been well acknowledged that cancer patients may present aberrant changes in the $\mathrm{T}$ lymphocyte subsets in the peripheral blood, which was reported to be related to the immune response imbalance due to imbalance of $\mathrm{CD} 3+, \mathrm{CD} 4+$, and CD8+ cells upon onset of malignant lesions, particularly the disturbance of the CD4+/CD8+ balance $[28,33]$. In this study, flow cytometry was used to analyze the $\mathrm{T}$ lymphocyte subsets and NK cells in the peripheral blood in late-stage NSCLC patients. Our results indicated that the number of $\mathrm{CD} 3+$ and $\mathrm{CD} 3+\mathrm{CD} 4+$ cells in the total $\mathrm{T}$ lymphocytes was significantly decreased in the peripheral blood of IV stage NSCLC patients compared with the healthy control. Additionally, the ratio of Tregs in the patients was obviously elevated compared with the healthy control. Taken together, we concluded that immune function disorder may present in the late-stage NSCLC patients, which was consistent with the previous descriptions [28, 32-34]. In particular, the decrease of $\mathrm{CD} 3+, \mathrm{CD} 3+\mathrm{CD} 4+$, and $\mathrm{NK}$ cell number and elevation of Tregs in the patients at stage IV was severe compared with these at stage III, which implied that the immune function disorder may be related to the clinical staging and distal metastasis. These findings suggested the presence of immunosuppression in the NSCLC patients, together with a decrease in the recognition and killing efficiency by the immune system towards the cancer cells, which resulted in the growth and metastasis of cancer cells. On this basis, it is reasonable to conclude that the immune function in the late-stage NSCLC patients is severely damaged, which hampers the recognition and killing of cancer cells by the host and triggers the extensive dissemination of cancer cells accordingly.

In order to investigate the correlation between the CTC number and the immunocyte distribution or clinicalpathological features, correlation analyses were performed between CTC number and distribution of immunocytes. Our data showed CTC number was positively correlated with the ratio of $\mathrm{CD} 3+, \mathrm{CD} 3+\mathrm{CD} 4+, \mathrm{CD} 4+/ \mathrm{CD} 8+$, and percentage of NK cells, while it was negatively correlated with the ratio of Tregs. Multiple regression analysis showed CTC positive rate was correlated with the staging and distal metastasis, as well as immunocyte status in the peripheral blood. Besides, several factors have been identified as the risk factors for CTC positivity, including low CD3+ percentage, $\mathrm{CD} 3+\mathrm{CD} 4+$, and $\mathrm{NK}$ cells, as well as high Treg ratio. This indicated that the number of effector $\mathrm{T}$ cells showed persistent decrease with the disease progression, while the number of immunosuppressive cells was gradually increased. The balance of $\mathrm{T}$ lymphocyte subsets was disrupted with the disease progression together with inhibition of immune function, which resulted in the escape of cancer cells disseminated in the peripheral blood from the immune surveillance. Therefore, our results confirmed the CTCs were closely related to the aberrant distribution of $\mathrm{T}$ lymphocyte subsets in the peripheral blood in late-stage NSCLC patients.

In conclusion, we firstly investigated the correlation between CTCs and the aberrant distribution of T lymphocyte subsets in the peripheral blood in late-stage NSCLC patients. Multivariate analysis indicated differentiation, NSCLC stage, and percentages of CD3+CD4+ cells, Tregs, and NK cells were the independent risk factors for CTCs. In the future, further prospective studies are needed to investigate the correlation between CTCs and the immunocyte number and function.

\section{Abbreviation \\ (CTCs): Circulating tumor cells \\ (NSCLC): Non-small-cell lung cancer \\ (Tregs): Regulatory T cells \\ (AJCC): American Joint Committee on Cancer \\ (PBMCs): Peripheral blood mononuclear cells \\ (SEM): Standard error of the mean.}

\section{Conflicts of Interest}

The authors declare that they have no conflicts of interest.

\section{Authors' Contributions}

Liang Ye and Fang Zhang contributed equally to this work.

\section{Acknowledgments}

This study was supported by the National Natural Science Foundation of China (no. 81570025).

\section{References}

[1] J. Ferlay, H. R. Shin, F. Bray, D. Forman, C. Mathers, and D. M. Parkin, "Estimates of worldwide burden of cancer in 2008: GLOBOCAN 2008," International Journal of Cancer, vol. 127, no. 12, pp. 2893-2917, 2010.

[2] R. Siegel, J. Ma, Z. Zou, and A. Jemal, "Cancer statistics, 2014," CA: a Cancer Journal for Clinicians, vol. 64, no. 1, pp. 9-29, 2014.

[3] D. Hanahan and R. A. Weinberg, "Hallmarks of cancer: the next generation," Cell, vol. 144, no. 5, pp. 646-674, 2011.

[4] C. R. Kelsey, L. B. Marks, D. Hollis et al., "Local recurrence after surgery for early stage lung cancer: an 11-year experience with 975 patients," Cancer, vol. 115, no. 22, pp. 5218-5227, 2009.

[5] R. M. Bremnes, T. Donnem, S. Al-Saad et al., "The role of tumor stroma in cancer progression and prognosis: emphasis on carcinoma-associated fibroblasts and non-small cell lung cancer," Journal of Thoracic Oncology, vol. 6, no. 1, pp. 209-217, 2011.

[6] S. Maheswaran and D. A. Haber, "Circulating tumor cells: a window into cancer biology and metastasis," Current Opinion in Genetics \& Development, vol. 20, no. 1, pp. 96-99, 2010. 
[7] J. D. O'Flaherty, S. Gray, D. Richard et al., "Circulating tumour cells, their role in metastasis and their clinical utility in lung cancer," Lung Cancer, vol. 76, no. 1, pp. 19-25, 2012.

[8] M. Ignatiadis, M. Lee, and S. S. Jeffrey, "Circulating tumor cells and circulating tumor DNA: challenges and opportunities on the path to clinical utility," Clinical Cancer Research, vol. 21, no. 21, pp. 4786-4800, 2015.

[9] M. G. Krebs, R. Sloane, L. Priest et al., "Evaluation and prognostic significance of circulating tumor cells in patients with non-small-cell lung cancer," Journal of Clinical Oncology, vol. 29, no. 12, pp. 1556-1563, 2011.

[10] S. I. Grivennikov, F. R. Greten, and M. Karin, "Immunity, inflammation, and cancer," Cell, vol. 140, no. 6, pp. 883-899, 2010.

[11] O. J. Finn, "Cancer immunology," New England Journal of Medicine, vol. 358, no. 25, pp. 2704-2715, 2008.

[12] L. de la Cruz-Merino, E. Grande-Pulido, A. Albero-Tamarit, and M. E. Codes-Manuel de Villena, "Cancer and immune response: old and new evidence for future challenges," Oncologist, vol. 13, no. 12, pp. 1246-1254, 2008.

[13] S. Wernersson and G. Pejler, "Mast cell secretory granules: armed for battle," Nature Reviews Immunology, vol. 14, no. 7, pp. 478-494, 2014.

[14] J. Pahl and A. Cerwenka, "Tricking the balance: NK cells in anti-cancer immunity," Immunobiology, vol. 222, no. 1, pp. 11-20, 2017.

[15] P. Deepak and A. Acharya, "Anti-tumor immunity and mechanism of immunosuppression mediated by tumor cells: role of tumor-derived soluble factors and cytokines," International Reviews of Immunology, vol. 29, no. 4, pp. 421-458, 2010.

[16] S. Durowicz, W. L. Olszewski, J. Dluzniewska, D. Laszuk, and B. Lukomska, "Cytotoxic sinusoidal lymphocytes of liver transplant are effective against circulating tumor cells," Transplantation Proceedings, vol. 31, no. 1-2, pp. 825-827, 1999.

[17] M. Mego, H. Gao, E. N. Cohen et al., "Circulating tumor cells (CTC) are associated with defects in adaptive immunity in patients with inflammatory breast cancer," Journal of Cancer, vol. 7, no. 9, pp. 1095-1104, 2016.

[18] J. M. Reuben, B. N. Lee, C. Li et al., "Biologic and immunomodulatory events after CTLA- 4 blockade with ticilimumab in patients with advanced malignant melanoma," Cancer, vol. 106, no. 11, pp. 2437-2444, 2006.

[19] H. Qiu, W. Xiao-Jun, Z. Zhi-Wei et al., "The prognostic significance of peripheral T-lymphocyte subsets and natural killer cells in patients with colorectal cancer," HepatoGastroenterology, vol. 56, no. 94-95, pp. 1310-1315, 2009.

[20] F. Tanaka, K. Yoneda, N. Kondo et al., "Circulating tumor cell as a diagnostic marker in primary lung cancer," Clinical Cancer Research, vol. 15, no. 22, pp. 6980-6986, 2009.

[21] D. Chudasama, A. Rice, G. Soppa, and V. Anikin, "Circulating tumour cells in patients with lung cancer undergoing endobronchial cryotherapy," Cryobiology, vol. 71, no. 1, pp. 161-163, 2015.

[22] A. Alama, A. Truini, S. Coco, C. Genova, and F. Grossi, "Prognostic and predictive relevance of circulating tumor cells in patients with non-small-cell lung cancer," Drug Discovery Today, vol. 19, no. 10, pp. 1671-1676, 2014.

[23] J. F. Dorsey, G. D. Kao, K. M. MacArthur et al., "Tracking viable circulating tumor cells (CTCs) in the peripheral blood of non-small cell lung cancer (NSCLC) patients undergoing definitive radiation therapy: pilot study results," Cancer, vol. 121, no. 1, pp. 139-149, 2015.

[24] J. M. Hou, M. Krebs, T. Ward et al., "Circulating tumor cells as a window on metastasis biology in lung cancer," The American Journal of Pathology, vol. 178, no. 3, pp. 989-996, 2011.

[25] A. Gabrielson, Y. Wu, H. Wang et al., "Intratumoral CD3 and CD8 T-cell densities associated with relapse-free survival in HCC," Cancer Immunology Research, vol. 4, no. 5, pp. 419-430, 2016.

[26] M. G. Morvan and L. L. Lanier, "NK cells and cancer: you can teach innate cells new tricks," Nature Reviews Cancer, vol. 16, no. 1, pp. 7-19, 2016 .

[27] C. Zhang, H. Xin, W. Zhang et al., "CD5 binds to interleukin-6 and induces a feed-forward loop with the transcription factor STAT3 in B cells to promote cancer," Immunity, vol. 44, no. 4, pp. 913-923, 2016.

[28] D. Wolf, S. Sopper, A. Pircher, G. Gastl, and A. M. Wolf, "Treg(s) in cancer: friends or foe?," Journal of Cellular Physiology, vol. 230, no. 11, pp. 2598-2605, 2015.

[29] J. P. Cerliani, T. Dalotto-Moreno, D. Compagno et al., "Study of galectins in tumor immunity: strategies and methods," Methods in Molecular Biology, vol. 1207, pp. 249-268, 2015.

[30] I. Mellman, G. Coukos, and G. Dranoff, "Cancer immunotherapy comes of age," Nature, vol. 480, no. 7378, pp. 480-489, 2011.

[31] K. C. Ohaegbulam, A. Assal, E. Lazar-Molnar, Y. Yao, and $\mathrm{X}$. Zang, "Human cancer immunotherapy with antibodies to the PD-1 and PD-L1 pathway," Trends in Molecular Medicine, vol. 21, no. 1, pp. 24-33, 2015.

[32] N. Erfani, S. M. Mehrabadi, M. A. Ghayumi et al., "Increase of regulatory $\mathrm{T}$ cells in metastatic stage and CTLA- 4 over expression in lymphocytes of patients with non-small cell lung cancer (NSCLC)," Lung Cancer, vol. 77, no. 2, pp. 306-311, 2012.

[33] W. J. Wang, Z. Tao, W. Gu, and L. H. Sun, "Variation of blood T lymphocyte subgroups in patients with non- small cell lung cancer," Asian Pacific Journal of Cancer Prevention, vol. 14, no. 8, pp. 4671-4673, 2013.

[34] L. J. Wesselius, D. L. Wheaton, L. J. Manahan-Wahl, S. L. Sherard, S. A. Taylor, and N. A. Abdou, "Lymphocyte subsets in lung cancer,” Chest, vol. 91, no. 5, pp. 725-729, 1987. 


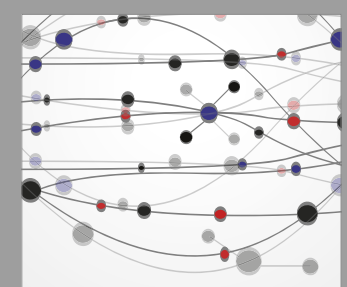

The Scientific World Journal
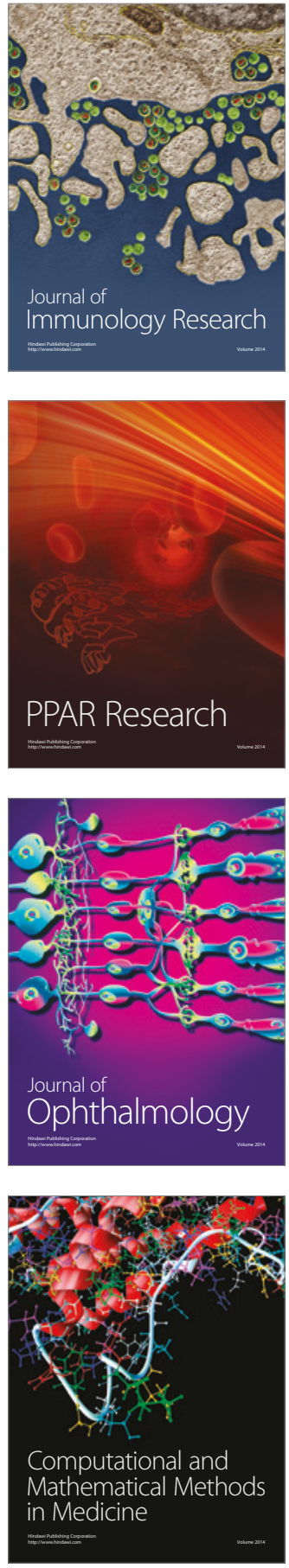

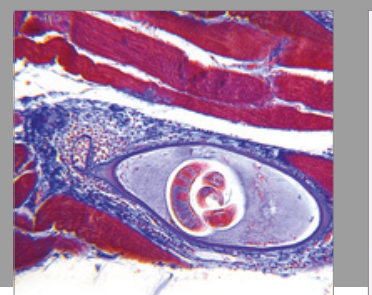

Gastroenterology Research and Practice
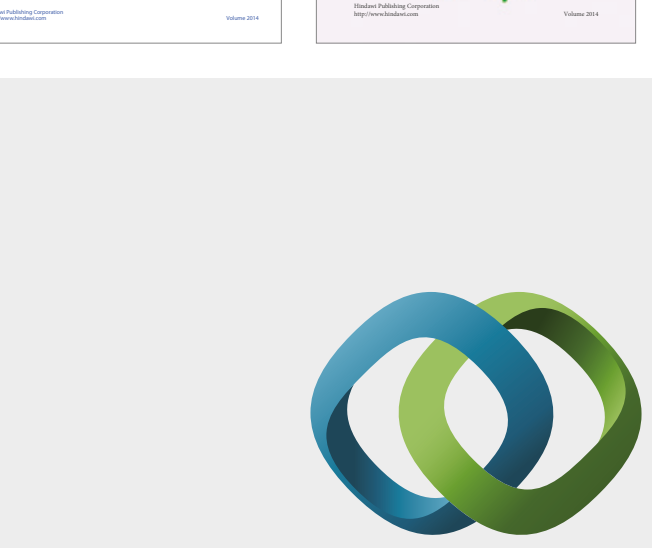

\section{Hindawi}

Submit your manuscripts at

https://www.hindawi.com
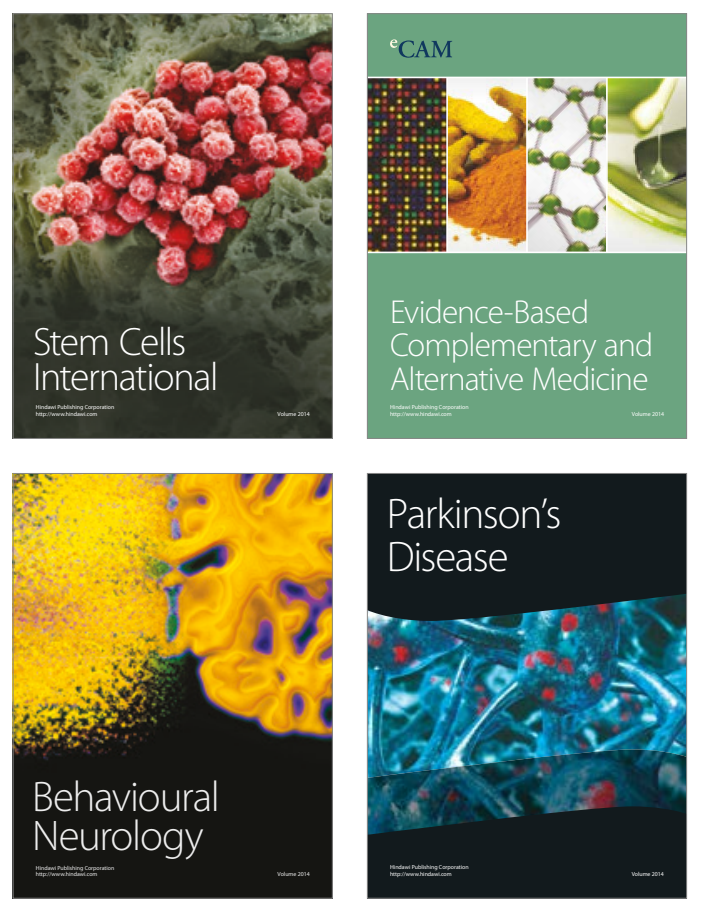
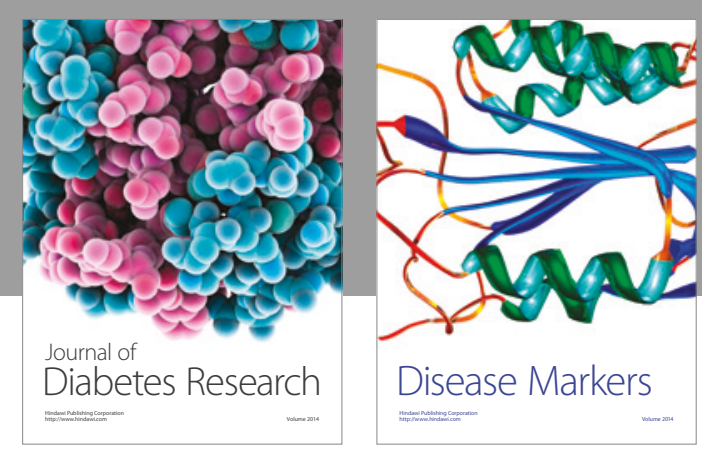

Disease Markers
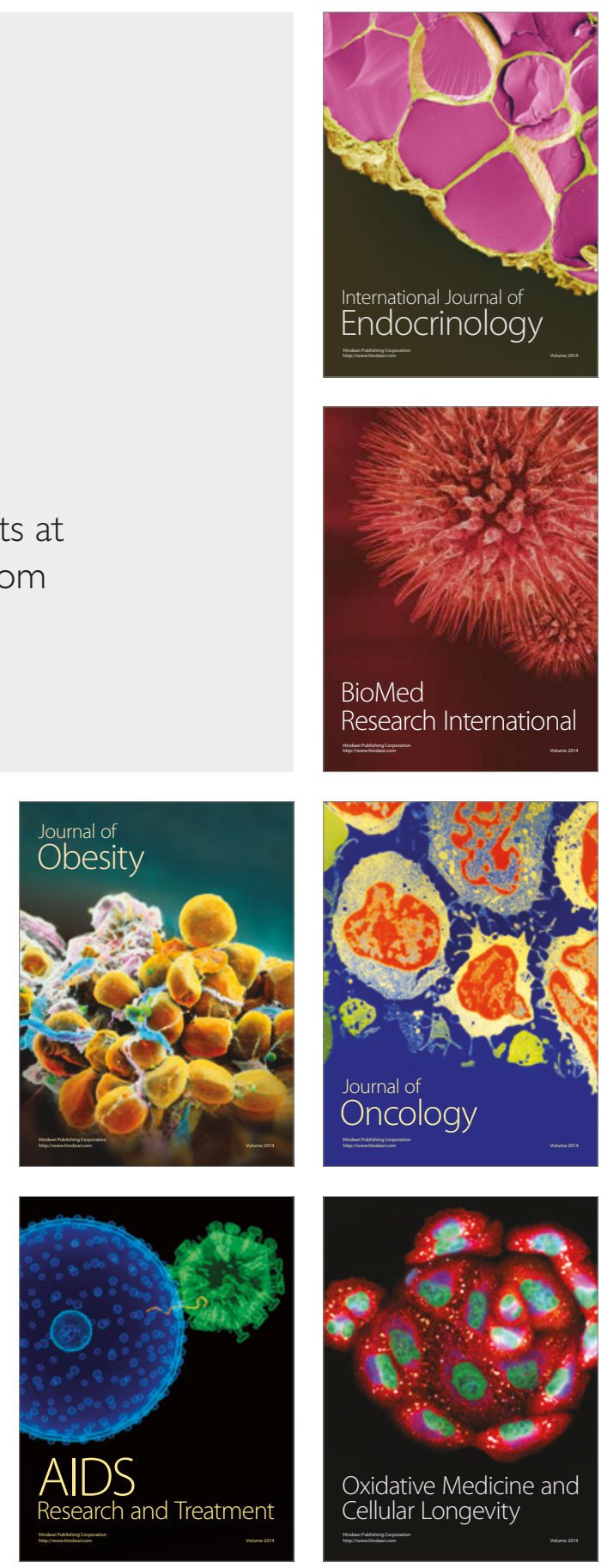